\title{
How Data Dependent is a Nonlinear Subdivision Scheme? - A Case Study Based on Convexity Preserving Subdivision
}

\author{
Thomas Pok-Yin $\mathrm{Yu}$ \\ Department of Mathematics, Drexel University \\ 3141 Chestnut Street, 206 Korman Center \\ Philadelphia, PA 19104 \\ Email: yut@drexel.edu
}

April 2, 2005

Revised: November 8, 2005

\begin{abstract}
:
The regularity of the limit function of a linear subdivision scheme is essentially irrelevant to the initial data. How data dependent, then, is the regularity of the limit of a nonlinear subdivision scheme? The answer is the most obvious it depends. In this paper, we prove that the nonlinear convexity preserving subdivision scheme developed independently by Floater/Micchelli [12] and Kuijt/van Damme [14] exhibits a rather strong nonlinear, data-dependent, behavior: For any $\nu \in(1,2)$, there exists initial convex data such that the critical Hölder regularity of the limit curve is exactly $\nu$. We also show that the limit function of any initial data always has Hölder regularity less than 2, unless if restricted to a subset of the domain at which the initial data is sampled from the convex branch of a rational polynomial of degree 2 over degree 1.

This result stands in contrast to what are reported in several recent publications on nonlinear subdivision schemes [18,21,20,5,22], in which various families of nonlinear subdivision schemes are either proved or empirically observed to have rather weak nonlinearity in the sense that they produce limit curves with smoothness insensitive to initial data.
\end{abstract}

Keywords. Subdivision/Refinement scheme, Nonlinear subdivision scheme, Convexity preserving subdivision, Hölder regularity, Homogeneous map, Real projective plane

Acknowledgements. The work of this research was partially supported by a NSF CAREER Award (CCR 9984501.) The author thanks Sinan Güntürk for discussions on dynamical systems. The research was partially finished while the author was visiting the Institute for Mathematical Sciences, National University of Singapore in August 2004. The visit was supported by the Institute.

Dedication: To the memory of Wong Suk Ling (1935-2004)

Mathematics Subject Classification. 26A15, 26A16, 26A18, 41A05, 42C40 


\section{Introduction}

Subdivision is a method for taking coarsely described data and recursively generating (typically smooth) data at finer and finer resolution. It can be used to rapidly generate curves and surfaces with built-in multiple level-of-details. Linear subdivision in the regular grid setting is also well-known to be connected to wavelet construction via the MRA framework due to Mallat and Meyer; and this connection had been explored and exploited in various nonlinear settings $[8,17,2,18,5]$ to construct various nonlinear 'wavelet' transforms. Despite the interests in both wavelets and computer-aided geometric design, there has been little theory on nonlinear subdivision schemes.

In recent years, it was either observed empirically or proved that certain nonlinear subdivision schemes exhibit the following weakly nonlinear property: for 'most' initial data, the limit curve produced by the nonlinear subdivision scheme has a critical Hölder regularity exactly the same as that produced by a related linear subdivision scheme. Notable examples include (i) median- and $p$-mean-interpolating subdivision schemes $[21,20,8,17,16]$, (ii) refinement schemes of manifold-valued data [18, 19, 22], and (iii) refinement schemes arising from normal multiresolution analysis [5]. A conceptually related discovery is reported in [3], where it is shown that an irregular grid variant of Dubuc's 4-point interpolatory subdivision scheme [9] has the exact same critical Hölder regularity as that in the regular grid setting, so long as the irregularity of the successively refined grids is somehow controlled; and it is conjectured $[3,23]$ that a similar phenomenon holds for a wilder class of irregular grid subdivision schemes.

Since the regularity of the limit function of a linear subdivision scheme is essentially irrelevant to the initial data, the aforementioned weakly nonlinear subdivision schemes share the same property of dataindependence.

In this paper, we show that the nonlinear convexity preserving subdivision scheme $(2.1)$ by $[14,12]$ produces limit curves with critical Hölder exponent quite heavily dependent on the initial data, unlike the behavior of a linear or weakly nonlinear subdivision scheme. Note that the convexity preserving subdivision scheme when applied to strictly convex data is simply based on the harmonic mean and, similar to the aforementioned weakly nonlinear schemes, may not occur to be data dependent at first glance. There exist nonlinear refinement schemes that are more data adaptive in appearance, e.g. the edge adapted or ENO refinement schemes in $[2,7]$.

While this paper is intended to be self-contained, the proof of our main result (Theorem 2.1) uses a key idea from [21], which the latter is described by the commutative diagram in Figure 1.

We reiterate the lame statement that a lot is known about linear subdivision, but little is known about their nonlinear counterparts; there are currently many unsolved open questions in the nonlinear subdivision literature, see the non-exhaustive list: $[18,19,5,21,20,8,17,13,16,15,14,12,2,22]$. Subdivision schemes in various geometric and nonlinear settings are of recent practical interests because of their natural connection with multiscale representations of different data types.

\section{Convexity Preserving Subdivision}

In $[14,12]$, the following nonlinear subdivision scheme is introduced: $f_{j+1}=S f_{j}$ where $S: \ell(\mathbb{Z}) \rightarrow \ell(\mathbb{Z})$ is defined by

$$
f_{j+1,2 k}=f_{j, k}, \quad f_{j+1,2 k+1}=\frac{f_{j, k}+f_{j, k+1}}{2}-\frac{1}{8} H\left(\left(\triangle^{2} f_{j}\right)_{k-1},\left(\triangle^{2} f_{j}\right)_{k}\right) .
$$

Here $\Delta$ is forward difference operator, and $H(\cdot, \cdot)$ denotes harmonic mean, i.e. $H(a, b)=2 a b /(a+b)$ if $a b>0$, and we define $H(a, b)=0$ if $a b \leqslant 0$.

It is helpful to bear in mind the linear counterpart of (2.1) based on replacing the nonlinear harmonic 
mean by the linear arithmetic mean:

$$
\begin{aligned}
f_{j+1,2 k}=f_{j, k}, \quad f_{j+1,2 k+1} & =\frac{f_{j, k}+f_{j, k+1}}{2}-\frac{1}{8} \text { Average }\left(\left(\triangle^{2} f_{j}\right)_{k-1},\left(\triangle^{2} f_{j}\right)_{k}\right) \\
& =\frac{9}{16}\left(f_{j, k}+f_{j, k+1}\right)-\frac{1}{16}\left(f_{j, k-1}+f_{j, k+2}\right) .
\end{aligned}
$$

This is the well-known subdivision scheme by Dubuc [9]; we denote its subdivision operator by $\bar{S}: \ell(\mathbb{Z}) \rightarrow$ $\ell(\mathbb{Z})$.

For $r=1,2$, there exist (nonlinear) subdivision operators $S^{[r]}$ such that

$$
S^{[r]} \circ \Delta^{r}=\Delta^{r} \circ S .
$$

In particular, $S^{[2]} \Delta^{2} v=\Delta^{2} S v$; if we write $w=\Delta^{2} v$, we have

$$
\begin{aligned}
&\left(S^{[2]} w\right)_{2 k}=\left(\Delta^{2} S v\right)_{2 k}=(S v)_{2 k}-2(S v)_{2 k+1}+(S v)_{2 k+2} \\
&= v_{k}-2\left[\frac{v_{k}+v_{k+1}}{2}-\frac{1}{8} H\left(\left(\triangle^{2} v\right)_{k-1},\left(\triangle^{2} v\right)_{k}\right)\right]+v_{k+1} \\
&= \frac{1}{4} H\left(w_{k-1}, w_{k}\right), \\
&\left(S^{[2]} w\right)_{2 k+1}=\left(\Delta^{2} S v\right)_{2 k+1}=(S v)_{2 k+1}-2(S v)_{2 k+2}+(S v)_{2 k+3} \\
&= \frac{v_{k}+v_{k+1}}{2}-\frac{1}{8} H\left(\left(\triangle^{2} v\right)_{k-1},\left(\triangle^{2} v\right)_{k}\right)-2 v_{k+1}+ \\
& \frac{v_{k+1}+v_{k+2}}{2}-\frac{1}{8} H\left(\left(\triangle^{2} v\right)_{k},\left(\triangle^{2} v\right)_{k+1}\right) \\
&=\frac{w_{k}}{2}-\frac{1}{8}\left[H\left(w_{k-1}, w_{k}\right)+H\left(w_{k}, w_{k+1}\right)\right] .
\end{aligned}
$$

As a comparison, there exist linear subdivision operators $\bar{S}^{[r]}, r=1,2,3,4$, such that $\bar{S}^{[r]} \circ \Delta^{r}=\Delta^{r} \circ \bar{S}$.

It is easy to check, using (2.4)-(2.5), that $S^{[2]}$ is positivity preserving, consequently $S^{[1]}$ is monotonicity preserving and $S$ is convexity preserving. These properties of $S$ are not shared by its linear counterpart $\bar{S}$. More in-depth discussions of the relationships among convexity preservation, nonlinear means and rational interpolation can be found in $[14,12,11]$. We shall later use the fact that $S$ reproduces rational polynomials of degree 2 over degree 1.

We denote by $\mathbb{R}_{+}$the set of positive real numbers, and let $\ell_{+}(\mathbb{Z}):=\left\{v \mid v: \mathbb{Z} \rightarrow \mathbb{R}_{+}\right\}$, and $\ell_{+}^{\infty}(\mathbb{Z}):=$ $\ell_{+}(\mathbb{Z}) \cap \ell^{\infty}(\mathbb{Z})$. By the positivity preserving and locality properties of $S^{[2]}$, we can view it as operators on either $\ell_{+}(\mathbb{Z})$ or $\ell_{+}^{\infty}(\mathbb{Z})$.

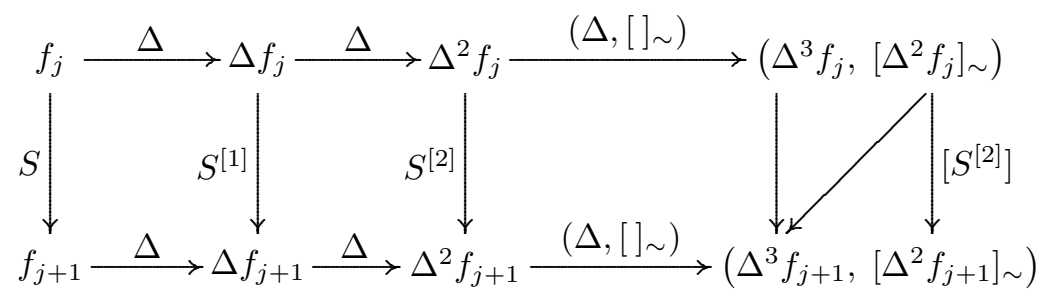

Figure 1: Commutation relations for $S$.

By (2.4)-(2.5), we have

$$
\left(S^{[2]} w\right)_{2 k, 2 k+1,2 k+2}=D\left(w_{k-1}, w_{k}, w_{k+1}\right),
$$

where the nonlinear map $D: \mathbb{R}^{3} \rightarrow \mathbb{R}^{3}$ is given by

$$
D\left(w_{1}, w_{2}, w_{3}\right)=\left(\frac{H\left(w_{1}, w_{2}\right)}{4}, \frac{w_{2}}{2}-\frac{1}{8}\left(H\left(w_{1}, w_{2}\right)+H\left(w_{2}, w_{3}\right)\right), \frac{H\left(w_{2}, w_{3}\right)}{4}\right) .
$$


Since $D$ is homogeneous (i.e. $D(\lambda w)=\lambda D(w)$ for all $\lambda \in \mathbb{R}$ and $w \in \mathbb{R}^{3}$ ), it induces a quotient map $\pi: \mathbb{P}\left(\mathbb{R}^{3}\right) \rightarrow \mathbb{P}\left(\mathbb{R}^{3}\right)$ via the formula $\pi\left([x]_{\sim}\right)=[D x]_{\sim}$. Here if $V$ is a real vector space, $\mathbb{P}(V):=V / \sim:=$ $\left\{[v]_{\sim}: v \in V\right\}$ where $\sim$ is the equivalence relation defined by $v \sim v^{\prime} \Longleftrightarrow \exists c \neq 0$ such that $v=c v^{\prime}$.

Since $D$ leaves $\mathbb{R}_{+}^{3}$ invariant, so does $\pi$ to $\left\{[v]_{\sim}: v \in \mathbb{R}_{+}^{3}\right\}$. Clearly $\left\{[v]_{\sim}: v \in \mathbb{R}_{+}^{3}\right\}$ can be identified with $\mathbb{R}_{+}^{2}$ by pairing $[x, 1, y]_{\sim}^{T}$ with $(x, y)$ (here $x, y>0$.) Viewing $\pi$ as a map on $\mathbb{R}_{+}^{2}$ under this identification, we have, by (2.7),

$$
\pi(x, y)=\left(\frac{2 x(1+y)}{2+x+y}, \frac{2 y(1+x)}{2+x+y}\right)
$$

We have the following facts pertaining to this map:

[P1] $(x, x)$ is a fixed point of $\pi$ for any $x>0$.

[P2] For any $x, y>0$, there is a unique $\bar{x}>0$ such that $\lim _{n \rightarrow \infty} \pi^{n}(x, y)=(\bar{x}, \bar{x})$.

Proof: [P1] takes care of the case of $x=y$. Assume $x>y$, the other case is symmetrical. The observation that

$$
1<\frac{x y+x}{x y+y}=\frac{\pi(x, y)_{1}}{\pi(x, y)_{2}}=\frac{x}{y} \frac{1+y}{1+x}<\frac{x}{y}
$$

implies that as $n$ increases the first component of $\pi^{n}(x, y)$ decreases monotonically to a limit value $\bar{x}$, whereas the second component of $\pi^{n}(x, y)$ increases monotonically to the same value.

[P3] For any $0<a<b, \pi$ leaves the square $R:=[a, b] \times[a, b]$ invariant, i.e. $\pi(R) \subseteq R$.

Proof: Let $(x, y) \in R$. Without loss of generality, assume $x \geqslant y$. We seek to show that $\left(x^{\prime}, y^{\prime}\right):=\pi(x, y)$ continues to belong to $R$. By (2.9), we have (i) $x^{\prime} \leqslant x$, (ii) $y^{\prime} \geqslant y$ and (iii) $x^{\prime} \geqslant y^{\prime}$. Assume the contrary that $\left(x^{\prime}, y^{\prime}\right) \notin R$, then either $x^{\prime}<a$ or $y^{\prime}>b$. If $x^{\prime}<a$, then $y^{\prime}<a$ by (iii), so $y<a$ by (ii), contradicting the assumption that $(x, y) \in R$. A similar contradiction can be generated if $y^{\prime}>b$.

The following is a more quantitative version of [P2], and is due to Sinan Güntürk.

[P4] $L(x, y):=x y /(2+x+y)$ is invariant under $\pi$, i.e. $L(\pi(x, y))=L(x, y)$. Therefore, for any $\left(x^{\prime}, y^{\prime}\right) \in \mathbb{R}_{+}^{2}$, the orbit $\left\{\pi^{n}\left(x^{\prime}, y^{\prime}\right),: n=0,1,2, \ldots\right\}$ lies on the level curve $C=\left\{(x, y): L(x, y)=x^{\prime} y^{\prime} /\left(2+x^{\prime}+y^{\prime}\right)\right\}$ and converges (in a monotonic fashion as described in [P2]) to the limit point $(\bar{x}, \bar{x})$ where $\bar{x}$ is the positive root of the quadratic equation $L(\bar{x}, \bar{x})=x^{\prime} y^{\prime} /\left(2+x^{\prime}+y^{\prime}\right)$.

In Lemma 2.5 we show that the curve $x y /(2+x+y)=1 / 4$ corresponds exactly to data sampled from rational polynomials of degree 2 over degree 1 .

Let $\Delta: \ell(\mathbb{Z}) \rightarrow \ell(\mathbb{Z})$ or $\mathbb{R}^{N} \rightarrow \mathbb{R}^{N-1}(N \geqslant 2)$ be the forward differencing operator, defined by $(\Delta m)_{i}=m_{i+1}-m_{i}$. Let

$$
\Delta^{1}:=\Delta, \quad \Delta^{r+1}:=\Delta \circ \Delta^{r} .
$$

(Clearly we are abusing notations when $\Delta$ acts on finite vectors, in this case $\Delta^{r}$ maps $\mathbb{R}^{N}$ to $\mathbb{R}^{N-r}$ for $N \geqslant r+1$.)

Next we consider the shrinking factor: for $(x, y) \neq(1,1)$, define

$$
s(x, y):=\frac{\|\Delta D([x, 1, y])\|_{\infty}}{\|\Delta[x, 1, y]\|_{\infty}}=\frac{\max \left(\left|\frac{1}{2}-\frac{3}{8} H(1, x)-\frac{1}{8} H(1, y)\right|,\left|\frac{1}{2}-\frac{1}{8} H(1, x)-\frac{3}{8} H(1, y)\right|\right)}{\max (|1-x|,|1-y|)} .
$$

We define $s(1,1)=1 / 4$ in order to make $s(x, x)=[2(1+x)]^{-1}$ continuous on $x>0$. It is elementary to verify that

[S1] $s(x, y) \in(0,1 / 2)$ for $(x, y) \in \mathbb{R}_{+}^{2}$. 
[S2] $s(x, y)$ is discontinuous at (and only at) $(x, y)=(1,1)$. In a small neighborhood of $(1,1), s(x, y)$ ranges from a little below $1 / 8$ to a little above $1 / 4$; precisely one can check that

$$
\begin{aligned}
& \lim _{\varepsilon \rightarrow 0} \inf _{\|(x, y)-(1,1)\|<\varepsilon} s(x, y)=\frac{1}{8}=\lim _{\substack{(x, y) \rightarrow(1,1) \\
x y /(2+x+y)=1 / 4}} s(x, y), \\
& \lim _{\varepsilon \rightarrow 0} \sup _{\|(x, y)-(1,1)\|<\varepsilon} s(x, y)=\frac{1}{4}=\lim _{\substack{(x, y) \rightarrow(1,1) \\
x=y}} s(x, y) .
\end{aligned}
$$

See Figure 2. Warning: the 0.25-contour in Figure 2(b), which is exactly the curve $x y /(2+x+y)=1 / 4$, can be misleading due to the discontinuity at $(1,1)$ : if $\left(x_{0}, y_{0}\right)$ is any point on this curve not equal to $(1,1)$, then $\lim _{n \rightarrow \infty} s\left(\pi^{n}\left(x_{0}, y_{0}\right)\right)=1 / 8 \neq s\left(\lim _{n \rightarrow \infty} \pi^{n}\left(x_{0}, y_{0}\right)\right)=1 / 4$.

[S3] $s(x, y) \leqslant s(\pi(x, y))$ with equality holds iff $x=y$.

See also Figure 2. Combining [S3] with [P3] and that $s(x, x)$ is decreasing in $x$, we have

$$
\sup _{\theta \in[a, b] \times[a, b]} s(\theta)=s(a, a)=[2(1+a)]^{-1} .
$$

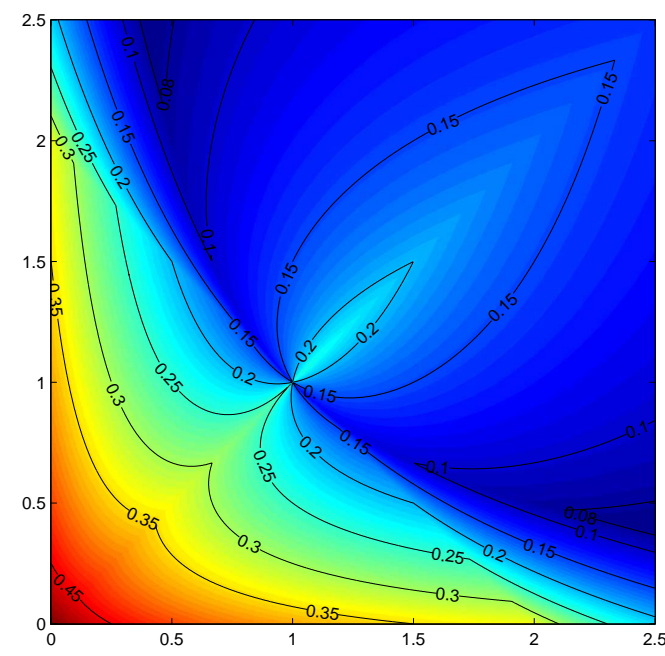

(a)
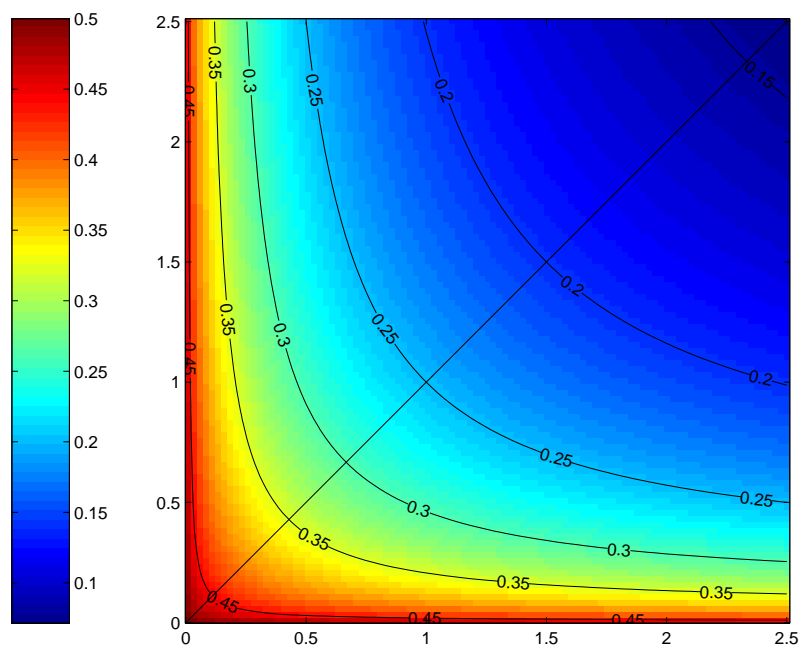

(b)

Figure 2: (a) Shrinking factor $s(x, y)$, (b) Asymptotic shrinking factor $s\left(\lim _{n \rightarrow \infty} \pi^{n}(x, y)\right)$.

Let $M, N$ be integers, $M<N$. It is well-known in approximation theory, see e.g. [1, 6], that for $f \in C([M, N])$, for $\alpha>0$, we have

$$
\begin{aligned}
f \in \operatorname{Lip} \alpha & \Longleftrightarrow \quad \exists r \in \mathbb{Z}_{+}, r>\alpha, \text { s.t. } \max _{2^{j} M \leqslant k \leqslant 2^{j} N-r}\left|\left(\Delta^{r} f_{j}\right)_{k}\right|=O\left(2^{-j \alpha}\right) \\
& \Longleftrightarrow \quad \forall r \in \mathbb{Z}_{+}, r>\alpha, \max _{2^{j} M \leqslant k \leqslant 2^{j} N-r}\left|\left(\Delta^{r} f_{j}\right)_{k}\right|=O\left(2^{-j \alpha}\right),
\end{aligned}
$$

where $f_{j}$ is the (length $2^{j}(N-M)+1$ ) sequence $\left(f_{j}\right)_{k}=f\left(2^{-j} k\right)$. This equivalence implies that the critical Hölder regularity exponent of $f$ can be determined from the exact asymptotic decay rate of $\max _{2^{j}} M \leqslant k \leqslant 2^{j} N-r$ $\left|\left(\Delta^{r} f_{j}\right)_{k}\right|$ for a large enough differencing order $r$, i.e.

$$
\sup \{\alpha: f \in \operatorname{Lip} \alpha\}=\sup \left\{\alpha: \max _{2^{j}}\left|\left(\Delta^{r} f_{j}\right)_{k}\right|=O\left(2^{-j \alpha}\right)\right\} .
$$

For continuous functions defined on the whole real line instead of a compact interval, the equivalence in (2.13) with $M=-\infty$ and $N=+\infty$ holds if we assume that $f$ is bounded. For a (possibly unbounded) 
continuous function $f: \mathbb{R} \rightarrow \mathbb{R}$, we say $f \in \operatorname{Lip} \alpha$ if $\left.f\right|_{[M, N]} \in \operatorname{Lip} \alpha$ for any $M<N$. Then, under the assumption that there exists $r>\alpha, r \in \mathbb{Z}_{+}$such that $\Delta^{r} f_{j} \in \ell^{\infty}$ for all $j$, we have

$$
f \in \operatorname{Lip} \alpha \Longleftrightarrow \max _{k \in \mathbb{Z}}\left|\left(\Delta^{r} f_{j}\right)_{k}\right|=O\left(2^{-j \alpha}\right) .
$$

Remark. Since $S$ is (point-)interpolatory, the subdivision data $S^{j} f_{0}$ is exactly the limit function $f$ sampled on the grid $2^{-j} \mathbb{Z}$, so the above result is directly applicable to analyzing the smoothness of $f$. For other subdivision schemes, linear or nonlinear, more subtle arguments related to stability are needed. See [21, Section 3] and the references therein.

As suggested by Figure 1, we shall use $r=3$ (i.e. 3rd order differences) to analyze the limit functions generated by the nonlinear convexity preserving scheme $S$.

A essential fact based on the locality property of $S$ is that if we specify the initial data $f_{0, k}$ at the integers $k=M-2, \ldots, N+2$, then the limit function restricted to the interval $[M, N]$ is uniquely determined. For $v \in \ell(\mathbb{Z})$, we denote by $S^{\infty} v$ or $f_{v}: \mathbb{R} \rightarrow \mathbb{R}$ the limit function; it is shown in [14] that $f_{v}$ is $C^{1}$ smooth for arbitrary strictly convex initial data $v$, i.e. $\Delta^{2} v \in \ell_{+}(\mathbb{Z})$ ).

Remark. Property [S1] already says that $\left\|\Delta^{3} S^{j} v\right\|_{\infty}=O\left((1 / 2)^{j}\right)$ if $\Delta^{2} v \in \ell_{+}^{\infty}(\mathbb{Z})$. With a refined argument, one can prove that

$$
\left\|\Delta^{3} S^{j} v\right\|_{\infty}=O\left((1 / 2+\epsilon)^{j}\right)
$$

for an $\epsilon>0$ dependent on $v$ and can be arbitrarily small. This, in turn, implies the above-mentioned $C^{1}$ result in [14].

Our main result is:

Theorem 2.1 Let $v \in \ell(\mathbb{Z})$ be such that $\left(\Delta^{2} v\right)_{i-1}=\left(\Delta^{2} v\right)_{i+1}$ and $\left(\Delta^{2} v\right)_{i}>0$ for all $i$. Assume

$$
\mu:=\frac{\left(\Delta^{2} v\right)_{2 i}}{\left(\Delta^{2} v\right)_{2 i-1}} \in(0,1) .
$$

(In particular, $\Delta^{2} v \in \ell_{+}^{\infty}(\mathbb{Z})$ and is a 2-periodic sequence.) Then

$$
\sup \left\{\alpha: f_{v} \in \operatorname{Lip} \alpha\right\}=\log _{2} 2(1+\mu) .
$$

Therefore, by adjusting the value $\mu \in(0,1)$, one can construct strictly convex initial data $v$ such that the limit function $f_{v}$ has a critical Hölder exponent equal to any value in $(1,2)$.

Proof: Write $f_{j}:=S^{j} v \in \ell(\mathbb{Z})$. As $r:=3>2>\log _{2} 2(1+\mu)$, it suffices to use $r=3$ in (2.14) and prove

$$
\left\|\Delta^{3} f_{j}\right\|_{\infty} \asymp[2(1+\mu)]^{-j}, \quad j \rightarrow \infty .
$$

Note that $s(x, x)=[2(1+x)]^{-1}$ according to $(2.10)$. The key point here is to relate the decay rate of $\left\|\Delta^{3} f_{j}\right\|_{\infty}$ to the maps $\pi: \mathbb{R}_{+}^{2} \rightarrow \mathbb{R}_{+}^{2}$ and $s: \mathbb{R}_{+}^{2} \rightarrow(0,1 / 2)$ introduced in (2.8) and (2.10).

$\left.1^{\circ}\right)$ Recall that $\Delta^{2} f_{j}=\left(S^{[2]}\right)^{j} w$, where $w:=\Delta^{2} v$. Recall also that the map $D: \mathbb{R}^{3} \rightarrow \mathbb{R}^{3}$ in (2.7) describes the operator $S^{[2]}$ via (2.6). Now, if we write

$$
\begin{aligned}
\mathbb{R}_{+}^{3} \ni w_{j, k} & :=\left(\left(S^{[2]}\right)^{j} w\right)_{k-2, k-1, k} \\
& =\left(\left(\Delta^{2} f_{j}\right)_{k-2},\left(\Delta^{2} f_{j}\right)_{k-1},\left(\Delta^{2} f_{j}\right)_{k}\right), \quad k=0, \ldots, 2^{j},
\end{aligned}
$$

then, in virtue of (2.6) we have:

$$
\begin{aligned}
w_{j+1,2 k} & =D\left(w_{j, k}\right), \quad k=0, \ldots, 2^{j}, \\
w_{j+1,2 k+1} & =\left[\left(w_{j+1,2 k}\right)_{2},\left(w_{j+1,2 k}\right)_{3}=\left(w_{j+1,2 k+2}\right)_{1},\left(w_{j+1,2 k+2}\right)_{2}\right]^{T}, \quad k=0, \ldots, 2^{j}-1 .
\end{aligned}
$$

Define $\theta_{j, k}=\left(\left(w_{j, k}\right)_{1},\left(w_{j, k}\right)_{3}\right) /\left(w_{j, k}\right)_{2} \in \mathbb{R}_{+}^{2}$. Then (2.19) gives

$$
\begin{aligned}
\theta_{j+1,2 k} & =\pi\left(\theta_{j, k}\right), \quad k=0, \ldots, 2^{j}, \\
\theta_{j+1,2 k+1} & =\Xi\left(\theta_{j+1,2 k}, \theta_{j+1,2 k+2}\right), \quad k=0, \ldots, 2^{j}-1,
\end{aligned}
$$


where $\Xi: \mathbb{R}_{+}^{2} \times \mathbb{R}_{+}^{2} \rightarrow \mathbb{R}_{+}^{2}, \Xi\left((x, y),\left(x^{\prime}, y^{\prime}\right)\right)=\left(1 / y, 1 / x^{\prime}\right)$. (See Figure 3.)

$2^{\circ}$ ) Under these notations, the assumption (2.15) of the theorem is equivalent to saying

$$
\theta_{0,0}=(\mu, \mu), \text { and } \theta_{0,1}=(1 / \mu, 1 / \mu),
$$

and (2.17) is, by symmetry of the data and the subdivision scheme, equivalent to

$$
\max _{k=0, \ldots, 2^{j}}\left\|\Delta w_{j, k}\right\|_{\infty} \asymp[2(1+\mu)]^{-j}, \quad j \rightarrow \infty .
$$

By the overlapping properties of $w_{j, k}$ 's, recall $(2.18)$, we do not need to use all the spatial indices $k$ for a given scale $j$, and (2.22) is equivalent to

$$
\max _{k=0, \ldots, 2^{j}}\left\|\Delta w_{j+1,2 k}\right\|_{\infty} \asymp[2(1+\mu)]^{-(j+1)}, \quad j \rightarrow \infty .
$$

Let $R_{\mu}:=[\mu, 1 / \mu] \times[\mu, 1 / \mu]$. It is clear that $\Xi\left(\theta, \theta^{\prime}\right) \in R_{\mu}$ if $\theta, \theta^{\prime} \in R_{\mu}$, together with property [P3] $\left(\pi\left(R_{\mu}\right) \subset R_{\mu}\right)$, we conclude that

$$
\theta_{j, k} \in R_{\mu}, \quad \forall j, k
$$

So by (2.12), we upper bound all the shrinking factors as:

$$
s\left(\theta_{j, k}\right) \leqslant s(\mu, \mu)=[2(1+\mu)]^{-1}, \quad \forall j, k .
$$

Notice also that

$$
\theta_{j, 0}=(\mu, \mu), \quad \forall j \geqslant 0 .
$$

Since

$$
\left\|\Delta w_{j+1,2 k}\right\|_{\infty}=s\left(\theta_{j, k}\right)\left\|\Delta w_{j, k}\right\|_{\infty}
$$

together with (2.25) we have

$$
\max _{k=0, \ldots, 2^{j}}\left\|\Delta w_{j+1,2 k}\right\|_{\infty}=O\left([2(1+\mu)]^{-(j+1)}\right) .
$$

On the other hand $\max _{k=0, \ldots, 2^{j}}\left\|\Delta w_{j+1,2 k}\right\|_{\infty}=\Omega\left([2(1+\mu)]^{-(j+1)}\right)$ since

$$
\max _{k}\left\|\Delta w_{j, k}\right\|_{\infty} \geqslant\left\|\Delta w_{j, 0}\right\|_{\infty}=\left\|\Delta w_{0,0}\right\|_{\infty} \prod_{l=1}^{j} s\left(\theta_{l, 0}\right) \stackrel{(2.26)}{=}\left\|\Delta w_{0,0}\right\|_{\infty} s(\mu, \mu)^{j} .
$$

So we have proved (2.17).

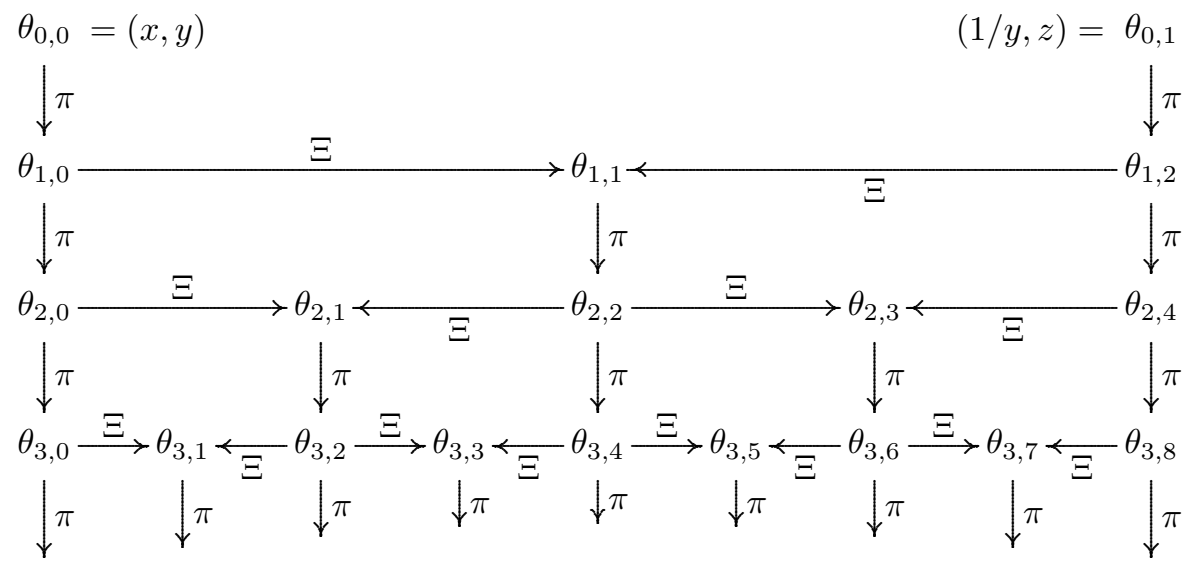

Figure 3: Recursive definition of $\theta_{j, k}$

In contrast to the nonlinear $S$, it is well-known that for Dubuc's scheme $\bar{S}$,

$$
\sup \left\{\nu: \bar{S}^{\infty} v \in \operatorname{Lip} \nu\right\}= \begin{cases}2, & \text { if }\left.v \in \ell(\mathbb{Z}) \backslash \Pi_{3}\right|_{\mathbb{Z}} \\ \infty, & \text { if }\left.v \in \Pi_{3}\right|_{\mathbb{Z}}\end{cases}
$$


In other words, except for initial data sampled from a polynomial of degree 3 or lower, the critical Hölder regularity of the limit curve is $2{ }^{1}$ This is characteristic of linear subdivision schemes and of the weakly nonlinear schemes mentioned in Section 1.

Note. Both (2.16) and (2.17) are not true if $\mu=1$. When $\mu=1, \Delta^{2} v$ is a constant sequence. By (2.4)-(2.5), $\Delta^{2} f_{j}=(1 / 4)^{j} \Delta^{2} v$, so $\Delta^{3} f_{j}$ is the zero sequence for all $j$. In this case, the limit function $f_{v}$ is a quadratic polynomial, which is infinitely smooth.

For general initial strictly convex data, we have the following:

Theorem 2.2 Let $w=\Delta^{2} v \in \ell_{+}(\mathbb{Z})$ and $k \in \mathbb{Z}$. Write

$$
x=\frac{w_{k-2}}{w_{k-1}}, \quad y=\frac{w_{k}}{w_{k-1}}, \quad z=\frac{w_{k+1}}{w_{k}} .
$$

1. If $\frac{x y}{2+x+y}=\frac{1}{4}=\frac{(1 / y) z}{2+(1 / y)+z}$, then $\left.S^{\infty} v\right|_{[k, k+1]}$ is a rational polynomial and is $C^{\infty}$;

2. Otherwise, for any $\varepsilon>0$,

$$
\sup \left\{\alpha:\left.S^{\infty} v\right|_{[k-\varepsilon, k+1+\varepsilon]} \in \operatorname{Lip} \alpha\right\}<2 .
$$

To prove this result we need the following facts pertaining to rational functions.

Remark 2.3 If $R(t)=\left(a t^{2}+b t+c\right) /(t-d)$, then $R^{\prime \prime}(t)=2\left(a d^{2}+b d+c\right) /(t-d)^{3}$. This means $R$ is convex on one side of the pole $t=d$ if and only if it is concave on the other side. We refer to the open subset of $\mathbb{R}$ $(\{t: t>d\}$ or $\{t: t<d\})$ at which $R^{\prime \prime}(t) \geqslant 0$ the convex branch of $R$. If $R(t)=a t^{2}+b t+c$, its convex branch is defined to be the whole of $\mathbb{R}$ when $a \geqslant 0$, and $\emptyset$ otherwise.

Remark 2.4 Let $t_{0}<t_{1}<\cdots<t_{n+1}$, and $f_{0}, f_{1}, \ldots, f_{n+1} \in \mathbb{R}$. Let $\left[t_{i}, \ldots, t_{k}\right] f$ be the divided difference of $f_{i}, \ldots, f_{k}$ at $t_{i}, \ldots, t_{k}$, defined, recursively, by

$$
\left[t_{\ell}\right] f:=f_{\ell}, \quad\left[t_{\ell}, \ldots, t_{\ell+n}\right] f:=\frac{\left[t_{\ell+1}, \ldots, t_{\ell+n}\right] f-\left[t_{\ell}, \ldots, t_{\ell+n-1}\right] f}{t_{\ell+n}-t_{\ell}}, \quad \text { if } n \geqslant 1 .
$$

If $D_{i}:=\left[t_{i}, t_{i+1}, \ldots, t_{i+n}\right] f, i=0,1$, are such that $D_{0} D_{1}>0$, then there is a unique rational polynomial $R(t)$ of degree $n$ over degree 1 that interpolates $f_{i}$ at $t_{i}$; moreover,

$$
R(t)=\frac{D_{1} \cdot\left(t_{n+1}-t\right) p_{0}(t)+D_{0} \cdot\left(t-t_{0}\right) p_{1}(t)}{D_{1} \cdot\left(t_{n+1}-t\right)+D_{0} \cdot\left(t-t_{0}\right)}
$$

where $p_{i}$ is the unique Lagrange interpolant of $f_{i}, \ldots, f_{i+n}$ at $t_{i}, \ldots, t_{i+n}, i=0,1$. Recall also Newton's formula

$$
p_{i}(t)=\sum_{k=i}^{i+n}\left[t_{k}, \ldots, t_{i+n}\right] f \prod_{\ell=k+1}^{i+n}\left(t-t_{\ell}\right) .
$$

Note that the numerator of $R(t)$ is a polynomial of degree $n$ or lower: by (2.30) the coefficient of $t^{n}$ in $p_{i}$ is $D_{i}$, so the coefficient of $t^{n+1}$ in the numerator of $R(t)$ is $D_{1} D_{0}-D_{0} D_{1}=0$. The denominator of $R(t)$ is a constant when $D_{0}=D_{1}$.

Note: When $D_{0} \neq D_{1}$, the rational function $(2.29)$ has a pole at $d=\left(t_{0} D_{0}-t_{n+1} D_{1}\right) /\left(D_{0}-D_{1}\right)$. The condition $D_{0} D_{1}>0$ guarantees that $d \neq t_{i}, i=0, \ldots, n+1$, as $D_{1}\left(t_{n+1}-t_{i}\right)+D_{0}\left(t_{i}-t_{0}\right)$ is positive (resp. negative) if both $D_{i}$ are positive (resp. negative.) However, an example would show that in general there is no guarantee that $d$ must stay outside the interval $\left[t_{0}, t_{n+1}\right]$. This last comment adds to the subtlety of the next lemma.

\footnotetext{
${ }^{1}$ More precisely: $f_{v}$ satisfies $\left|f_{v}^{\prime}(x+t)-f_{v}^{\prime}(x)\right|=O(t \log (1 / t))$ and this bound cannot be improved unless for data sampled from a cubic polynomial.
} 
Lemma 2.5 Let $f_{0}, f_{1}, \ldots, f_{4}$ be such that $w_{i}:=f_{i+2}-2 f_{i+1}+f_{i}>0$ for $i=0,1,2$. Set $x=w_{0} / w_{1}$ and $y=w_{2} / w_{1}$. Let $\bar{t} \in \mathbb{R}$ and $h>0$.

Then there exists a (unique) rational function $R(t)=\left(a t^{2}+b t+c\right) /(t-d)$ such that $R(\bar{t}+i h)=f_{i}$ for $i=0, \ldots, 4$ and $[\bar{t}, \bar{t}+4 h] \subset($ convex branch of $R$ ) if and only if

$$
\frac{x y}{2+x+y}=\frac{1}{4}
$$

Proof: Without loss of generality, we can assume $\bar{t}=0$ and $h=1$.

If $f_{i}=\left(a(i)^{2}+b(i)+c\right) /(i-d), i=0,1,2,3,4$, then $x=\left(f_{2}-2 f_{1}+f_{0}\right) /\left(f_{3}-2 f_{2}+f_{1}\right)=(-3+d) / d$, $y=\left(f_{4}-2 f_{3}+f_{2}\right) /\left(f_{3}-2 f_{2}+f_{1}\right)=(-1+d) /(-4+d)$. One can then verify (2.31) immediately. Notice that the assumption $[\bar{t}, \bar{t}+4 h] \subset$ (convex branch of $R$ ) is the same as saying $d<0$ or $d>4$, so there is no worry of division by zero in the definitions of $f_{i}$ 's.

To prove the converse, let

$$
D_{i}:=[i, i+1, i+2, i+3] f=\left(w_{i+1}-w_{i}\right) / 6, \quad i=0,1 .
$$

(We use the divided difference notation as mentioned in Remark 2.4.) Since $D_{0} D_{1}=\left(w_{1}-w_{0}\right)\left(w_{2}-w_{1}\right) / 36$, $D_{0} D_{1}>0$ follows from $(2.31)$ according to the following calculation: $\left(w_{1}-w_{0}\right)\left(w_{2}-w_{1}\right)=w_{1}(1-x)(y-1)=$ $w_{1}(1-x)((2+x) /(4 x-1)-1)=3 w_{1}(1-x)^{2} /(4 x-1)>0$. (Notice that $(2.31)$ implies that $x, y>1 / 4$.)

Knowing $D_{0} D_{1}>0$, Remark 2.4 then applies: The unique rational polynomial of degree 3 over degree 1 that interpolates $f_{0}, \ldots, f_{4}$ at $0, \ldots, 4$ is given by

$$
R(x)=\frac{D_{1}(4-t) p_{0}(t)+D_{0} t p_{1}(t)}{D_{1}(4-t)+D_{0} t} .
$$

The goal here is to the show that the numerator of the right-hand side of $(2.33)$ is in fact a polynomial of degree 2 or lower, and also to prove that the pole of $R$ is outside of the interval [0,4].

The coefficient of $t^{3}$ in the numerator of $R(x)$ is:

$$
\begin{array}{ll} 
& D_{1}\left(4\left(\text { coeff. of } t^{3} \text { in } p_{0}\right)-\left(\text { coeff. of } t^{2} \text { in } p_{0}\right)\right)+D_{0}\left(\text { coeff. of } t^{2} \text { in } p_{1}\right) \\
= & D_{1}\left(4 D_{0}-\left(w_{1} / 2-6 D_{0}\right)\right)+D_{0}\left(w_{2} / 2-9 D_{1}\right) \\
\stackrel{(2.32)}{=} & 36 w_{1}(2+x+y-4 x y) \stackrel{(2.31)}{=} 0 .
\end{array}
$$

The denominator is constant if $D_{0}=D_{1}$, in this case $x=y=1$ under the condition (2.31). Otherwise, $R$ has its pole at

$$
d=\frac{4 D_{1}}{D_{1}-D_{0}}=\frac{4(y-1)}{x+y-2} \stackrel{(2.31)}{=} \frac{3}{1-x},
$$

which stays out of the interval $[0,4]$ since (2.31) also implies $x>1 / 4$.

Proof of Theorem 2.2. Without loss of generality, assume $k=0$.

$1^{\circ}$ ) If $\frac{x y}{2+x+y}=\frac{1}{4}=\frac{(1 / y) z}{2+(1 / y)+z}$, then, by Lemma 2.5, there exists a unique rational polynomial $R(t)$ of degree 2 over degree 1 such that $f_{i}=R(i), i=-2, \ldots, 3$. It is known from [12] that the subdivision scheme reproduces $R$ on $[0,1]$, i.e. $\left.S^{\infty} v\right|_{[0,1]}=R$. The pole of $R$ is outside the interval $[-2,3]$. So $R$ is certainly $C^{\infty}$ on $[0,1]$.

$2^{\circ}$ ) To prove the second part of the theorem, we need to first recall the basic setup in part $1^{\circ}$ ) of the proof of Theorem 2.1. If we define $\theta_{j, k} k=0, \ldots, 2^{j}$ according to $(2.20)$ with

$$
\theta_{0,0}=(x, y), \quad \theta_{0,1}=(1 / y, z)
$$

(Recall also Figure 3.) We write $C:=\left\{(x, y) \in \mathbb{R}_{+}^{2}: x y /(2+x+y)=1 / 4\right\}, C^{+}:=\left\{(x, y) \in \mathbb{R}_{+}^{2}:\right.$ $x y /(2+x+y)>1 / 4\}$ and $C^{-}:=\left\{(x, y) \in \mathbb{R}_{+}^{2}: x y /(2+x+y)<1 / 4\right\}$. Note that $C^{+} \supset(1, \infty)^{2}$ and $C^{-} \supset(0,1)^{2}$. 
To prove the theorem, we claim that it suffices to show that

$$
\exists \bar{j}, \bar{k} \text { s.t. } \theta_{\bar{j}, \bar{k}} \in C^{-} .
$$

By properties [P2] and [P4] of $\pi$ and the identity $s(x, x)=[2(1+x)]^{-1},(2.34)$ is equivalent to saying

$$
\lim _{n \rightarrow \infty} s\left(\pi^{n}\left(\theta_{\bar{j}, \bar{k}}\right)\right)=s^{*}>1 / 4 .
$$

Now, recall from Part $1^{\circ}$ ) of the proof of Theorem 2.1 that

$$
s\left(\theta_{j, k}\right)=\frac{\max \left(\left|\left(\Delta^{3} f_{j+1}\right)_{2 k-2}\right|,\left|\left(\Delta^{3} f_{j+1}\right)_{2 k-1}\right|\right)}{\max \left(\left|\left(\Delta^{3} f_{j}\right)_{k-2}\right|,\left|\left(\Delta^{3} f_{j}\right)_{k-1}\right|\right)}
$$

and

$$
\theta_{j, k}=\left(\frac{\left(\Delta^{2} f_{j}\right)_{k-2}}{\left(\Delta^{2} f_{j}\right)_{k-1}}, \frac{\left(\Delta^{2} f_{j}\right)_{k}}{\left(\Delta^{2} f_{j}\right)_{k-1}}\right) .
$$

If $\theta_{j, k} \in C^{-}$, then at least one of its two components is strictly less than one, which in turn implies that the denominator of (2.36) is non-zero. So altogether (2.35) implies that

$$
\max _{-2 \leqslant k<2^{j}}\left|\left(\Delta^{3} f_{j}\right)_{k}\right|=\Omega\left(s_{-}^{j}\right)
$$

for any $s_{-} \in\left(1 / 4, s^{*}\right)$. Consequently, for any $\varepsilon>0,\left.S^{\infty} v\right|_{[-\varepsilon, 1+\varepsilon]}$ has Hölder regularity strictly less than 2 . $\left.3^{\circ}\right)$ So it remains to prove (2.34) under the assumption that either $(x, y) \notin C$ or $(1 / y, z) \notin C$.

There is nothing to prove if $(x, y) \in C^{-}$or $(1 / y, z) \in C^{-}$. So we can assume without loss of generality that

$$
\theta_{0,0}=(x, y) \in C^{+}, \quad \text { and } \quad \theta_{0,1}=(1 / y, z) \in C \cup C^{+} .
$$

As a warmup, let's first consider the case $y=1$. If $y=1$, then $1 / y=1, z \geqslant 1$ and $x>1$. But then $\theta_{1,0}=\pi((>1,1))=(>1,>1)$, and $\theta_{1,2}=\pi((1, \geqslant 1))=(\geqslant 1, \geqslant 1)$, so

$$
\theta_{1,1}=\Xi\left(\theta_{1,0}, \theta_{1,2}\right)=\left(\frac{1}{>1}, \frac{1}{\geqslant 1}\right)=(<1, \leqslant 1) \in C^{-},
$$

and we are done with the special case $y=1$.

We now consider the case $y<1$, the case of $y>1$ is similar.

Assume the contrary that no $\theta_{j, k} \in C^{-}$.

If $y<1$, then $x>1$, and $1 / y>1$, so we have

$$
\theta_{0,0}=(>1,<1), \quad \theta_{0,1}=(>1, *) .
$$

We know that $\theta_{1,0}=(>1, *), \theta_{1,2}=(>1, *)$. Observe that $\theta_{1,0}=(>1,<1)$, otherwise $\theta_{1,0}=(>1, \geqslant 1)$ and

$$
\theta_{1,1}=\Xi\left(\theta_{1,0}, \theta_{1,2}\right)=(1 /(\geqslant 1), 1 /(>1))=(\leqslant 1,<1) \in C^{-},
$$

violating our assumption. So we now have $\theta_{1,0}=(>1,<1), \theta_{1,1}=(>1, *)$. This brings us back to the same situation as in scale $j=0$, recall (2.37); this means, by induction,

$$
\theta_{n, 0}=(>1,<1), \quad \theta_{n, 1}=(>1, *), \quad \forall n .
$$

But this contradicts the fact that $\lim _{n \rightarrow \infty} \theta_{n, 0}=\lim _{n \rightarrow \infty} \pi^{n}\left(\theta_{0,0}\right)=(c, c), c>1$. This completes the proof of the theorem.

Remark. If $\theta_{0,0}, \theta_{0,1} \in C$, then all $\theta_{j, k}$ 's lie on $C$ as well, and are near $(1,1)$ for $j$ large enough. So, by $(2.11)$, the shrinking factors $s\left(\theta_{j, k}\right)$ are close to $1 / 8$ for large $j$, so $\left\|\Delta^{3} f_{j}\right\|_{\infty}$ decays essentially like $(1 / 8)^{j}=2^{-3 j}$. In this very case, we can only say that the critical Hölder regularity is no less than 3 , but 3 rd order differences maybe too "underpower" to determine the optimal Hölder regularity. In other words, the critical smoothness can be higher than 3 , this is exactly the case: Part 1 . of Theorem 2.2 says that the smoothness is $\infty$ (»3!) 


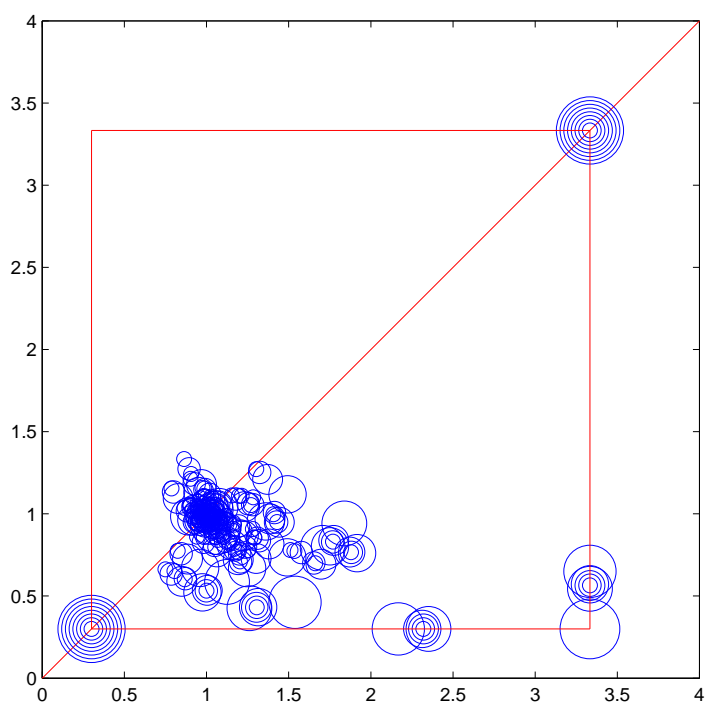

(a) $x=0.3$

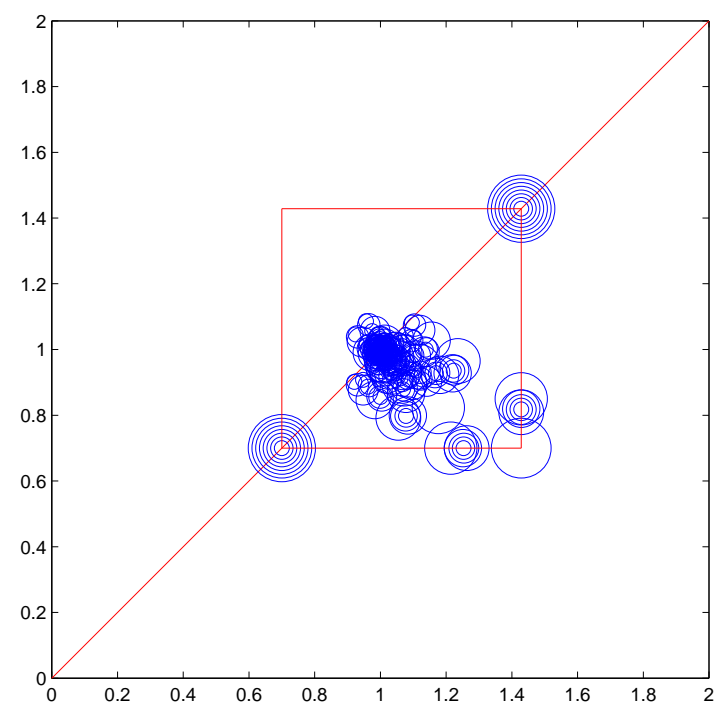

(b) $x=0.7$

Figure 4: For each $\theta_{j, k} \in \mathbb{R}_{+}^{2}$ defined by (2.20), a circle with center $\theta_{j, k}$ and radius which decreases linearly with $j$ is drawn. Notice that $\theta_{j, k}$ tends to cluster at $(1,1)$.

\section{Observations}

For linear subdivision schemes, it is typical that the local Hölder exponents at dyadic irrationals are higher than the global critical Hölder regularity, see [4, Section 4]. (Here we assume that the subdivision scheme is binary.) A similar property holds for the nonlinear convexity preserving scheme in this paper, except that it can be more easily explained than those linear examples in [4, Section 4]: while Theorem 2.2 says that the global regularity of the limit function $f_{v}$ for any initial data $v$ is less than 2, a classical result of A. D. Alexsandrov (see, e.g., [10]) asserts that $f_{v}$, being a convex function on $\mathbb{R}$, must be twice differentiable almost everywhere. (This also partly explains the observed clustering of $\theta_{j, k}$ about $(1,1)$ for arbitrary initial configuration $x, y, z>0$ (see e.g. Figure 4) - a fact that seems difficult to explain by elementary means.)

\section{References}

[1] Z. Ciesielski. Approximation of splines and its application to Lipschitz classes and to stochastic processes. In Approximation Theory of Functions, Proc. of the conference in Kaluga, 1975, pages 397-400. Nauka, Moscow, 1977.

[2] A. Cohen, N. Dyn, and B. Matei. Quasilinear subdivision schemes with applications to ENO interpolation. Applied and Computational Harmonic Analysis, 15(2):89-116, 2003.

[3] I. Daubechies, I. Guskov, and W. Sweldens. Regularity of irregular subdivision. Constructive Approximation, 15:381-426, 1999 .

[4] I. Daubechies and J. C. Lagarias. Two-scale difference equations II. Local regularity, infinite products of matrices and fractals. SIAM J. Math. Anal., 23(4):1031-1079, 1992.

[5] I. Daubechies, O. Runborg, and W. Sweldens. Normal multiresolution approximation of curves. Constructive Approximation, 3(4):399 - 463, 2004.

[6] Z. Ditzian. Moduli of smoothness using discrete data. J. Approx. Theory, 49:115-129, 1987.

[7] D. L. Donoho. Minimum entropy segmentation. In C.K. Chui et al, editor, Wavelets Theory, Algorithms and Applications, pages 233-269. Academic Press, 1994. 
[8] D. L. Donoho and T. P.-Y. Yu. Nonlinear pyramid transforms based on median-interpolation. SIAM Journal of Math. Anal., 31(5):1030-61, 2000.

[9] S. Dubuc. Interpolation through an iterative scheme. Journal of Mathematical Analysis and Applications, 114:185-204, 1986.

[10] L. C. Evans and R. F. Gariepy. Measure Theory and fine properties of functions. CRC Press, Boca Raton, Florida, 1992.

[11] M. S. Floater and C. A. Micchelli. Nonlinear means in geometric modeling. In N. K. Govil, R. N. Mohapatra, Z. Nashed, A. Sharma, and J. Szabados, editors, Approximation Theory: in Memory of A. K. Varma, pages 197-207. 1998.

[12] M. S. Floater and C. A. Micchelli. Nonlinear stationary subdivision. In N. K. Govil, R. N. Mohapatra, Z. Nashed, A. Sharma, and J. Szabados, editors, Approximation Theory: in Memory of A. K. Varma, pages 209-224. Marcel Dekker, New York, 1998.

[13] T. N. T. Goodman and T. P.-Y. Yu. Interpolation of medians. Advances in Computational Mathematics, 11(1):1-10, 1999.

[14] F. Kuijt and R. van Damme. Convexity preserving interpolatory subdivision schemes. Constructive Approximation, 14:609-630, 1998.

[15] P. Oswald. Smoothness of a nonlinear subdivision scheme. In A. Cohen, J.-L. Merrien, and L. L. Schumaker, editors, Curves and Surface Fitting: Saint-Malo 2002, pages 323-332. Nashboro Press, 2003.

[16] P. Oswald. Smoothness of nonlinear median-interpolation subdivision. Advances in Computational Mathematics, 20(4):401-423, 2004.

[17] J. S. Pang and T. P.-Y. Yu. Continuous M-estimators and their interpolation by polynomials. SIAM Journal of Numerical Analysis, 42(3):997 - 1017, 2004.

[18] I. Ur Rahman, I. Drori, V. C. Stodden, D. L. Donoho, and P. Schröder. Multiscale representations for manifold-valued data. SIAM Journal on Multiscale Modeling and Simulation, x(x):xxx-xxx, 2005.

[19] J. Wallner and N. Dyn. Convergence and $C^{1}$ analysis of subdivision schemes on manifolds by proximity. Computer Aided Geometric Design, 22(7):593-622, 2005.

[20] G. Xie and T. P.-Y. Yu. On a linearization principle for nonlinear $p$-mean subdivision schemes. In M. Neamtu and E. B. Saff, editors, Advances in Constructive Approximation, pages 519-533. Nasboro Press, 2004

[21] G. Xie and T. P.-Y. Yu. Smoothness analysis of nonlinear subdivision schemes of homogeneous and affine invariant type. Constructive Approximation, 22(2):219-254, 2005.

[22] T. P.-Y. Yu. Cutting corners on the sphere. Submitted for publication. Preprint available at http://www.rpi.edu/ yut/Papers/CuttingCorners.pdf, April 2005.

[23] T. P.-Y. Yu. On the regularity analysis of interpolatory Hermite subdivision schemes. Journal of Mathematical Analysis and Applications, 302(1):201-216, 2005. 